\title{
A WEIGHTED NORM INEQUALITY FOR FOURIER SERIES
}

\author{
BY RICHARD A. HUNT ${ }^{1}$ AND WO-SANG YOUNG
}

Communicated by Alberto Calderón, September 22, 1973

Let $M f(x)=\sup \left|S_{n} f(x)\right|$, where $S_{n} f$ denotes the $n$th partial sum of the Fourier series of $f$. We will show

$$
w \in A_{p}, p>1, \text { implies } \int[M f]^{p} w \leqq C \int|f|^{p} w .
$$

Recall that a nonnegative weight function $w \in A_{p}, p>1$, if there is a constant $K$ such that

$$
\left(\int_{I} w\right)\left(\int_{I} w^{-1 /(p-1)}\right)^{p-1} \leqq K|I|^{p}
$$

for all intervals $I$. The $A_{p}$ condition, $p>1$, characterizes all weights $w$ for which the mapping of $f$ into the Hardy-Littlewood maximal function of $f$ is bounded on the weighted $L^{p}$ space $L^{p}(w)$. (See Muckenhoupt [6].) This fundamental fact leads to boundedness on $L^{p}(w)$ for other operators which can be associated with the Hardy-Littlewood maximal function. For example, the conjugate function and more general singular integrals are of this type. Also, $\int\left|S_{n} f-f\right|^{p} w \rightarrow 0(n \rightarrow \infty)$ if and only if $w \in A_{p}$, $p>1$. (See Hunt, Muckenhoupt and Wheeden [5] and Coifman [3].) It follows that the inequality in (1) holds only if $w \in A_{p}, p>1$.

Our proof of (1) follows closely the proof in Coifman [3]. We will prove a Burkholder-Gundy type distribution function inequality which relates the weighted distribution functions of modified versions of $M f$ and the Hardy-Littlewood maximal function of $f$. (See Burkholder and Gundy [1].) To do this we will use the boundedness of $M$ on $L^{r}, r>1$, and an extremely useful consequence of the $A_{p}$ condition which relates the $w$-weighted measure and the Lebesgue measure of certain types of sets. This useful property is closely related to the development of Muckenhoupt [6] and was first explicitly used in connection with a distribution

AMS (MOS) subject classifications (1970). Primary 46E30; Secondary 42A20, 44A25.

${ }^{1}$ The research of the first-named author was supported in part by the National Science Foundation GP-18831. 
function inequality by Fefferman in an unpublished paper. The distribution function inequality implies the $L^{p}(w)$ norm of $M f$ is majorized by a constant multiple of the $L^{p}(w)$ norm of the modified Hardy-Littlewood maximal function of $f$. (1) then follows from Muckenhoupt's result on the $L^{p}\left(w^{\prime}\right)$ boundedness of the Hardy-Littlewood maximal function.

Let

$$
H_{r} f(x)=\sup _{h>0}\left(\frac{1}{2 h} \int_{|x-t|<h}|f(t)|^{r} d t\right)^{1 / r}, \quad r \geqq 1 .
$$

Note that $H_{1} f$ is the usual Hardy-Littlewood maximal function and so $\int\left[H_{1} f\right]^{s} w \leqq C \int|f|^{s} w$ if $w \in A_{s}, s>1$. (See Muckenhoupt [6].) Since $H_{r} f=\left(H_{1}\left(|f|^{r}\right)\right)^{1 / r}$, it follows that

$$
w \in A_{p / r}, r<p, \text { implies } \int\left[H_{r} f\right]^{p} w \leqq C \int|f|^{p} w .
$$

We will need to use (2) for some $r>1$. This is possible because of the following fundamental result of Muckenhoupt [6]:

$$
w \in A_{p}, p>1 \text {, implies } w \in A_{p / r} \text { for some } 1<r<p .
$$

Following Carleson [2], we replace $M f$ by

$$
M^{*} f=\sup _{n}\left|\int_{|x-t|<\pi} e^{-i n t} f(t) /(x-t) d t\right| .
$$

In fact, we will use

$$
M^{* *} f=\sup _{n} \sup _{\varepsilon>0}\left|\int_{\varepsilon<|x-t|<\pi} e^{-i n t} f(t) /(x-t) d t\right| .
$$

Standard arguments imply

(4) $M f \leqq C\left(H_{1} f+M^{*} f\right) \leqq C\left(H_{1} f+M^{* *} f\right) \leqq C\left(H_{1} f+H_{1}\left(M^{*} f\right)\right)$.

From (4) and (2) with $r=1$ we see that we may replace $M f$ by $M^{* *} f$ in (1). Also, since $\int\left[M^{*} f\right]^{r} \leqq C \int|f|^{r}, r>1$, (see Hunt [4]) we have

$$
r>1 \text { implies } \int\left[M^{* *} f\right]^{r} \leqq C \int|f|^{r} .
$$

Given $w \in A_{p}, p>1$, choose $r$ as in (3). We will prove

$$
m_{w}\left(M^{* *} f>3 \lambda, H_{r} f \leqq \gamma \lambda\right) \leqq C(\gamma) m_{w}\left(M^{* *} f>\lambda\right),
$$

where $C(\gamma) \rightarrow 0(\gamma \rightarrow 0) .\left(m_{w}(E)=\int_{E} w.\right)$

Given this weighted distribution function inequality it is easy to complete the proof of (1). From (6) we obtain

$$
m_{w}\left(M^{* *} f>3 \lambda\right) \leqq m_{w}\left(H_{r} f>\lambda \gamma\right)+C(\gamma) m_{w}\left(M^{* *} f>\lambda\right) .
$$


Hence,

$$
\begin{aligned}
p \int_{0}^{\infty} \lambda^{p-1} m_{w}\left(M^{* *} f>3 \lambda\right) d \lambda & \\
& \leqq p \int_{0}^{\infty} \lambda^{p-1} m_{w}\left(H_{r} f>\gamma \lambda\right) d \lambda+C(\gamma) p \int_{0}^{\infty} \lambda^{p-1} m_{w}\left(M^{* *} f>\lambda\right) d \lambda
\end{aligned}
$$

and so $\int\left[M^{* *} f\right]^{p} w \leqq\left[\gamma^{-p} /\left(3^{-p}-C(\gamma)\right)\right] \int\left[H_{r} f\right]^{p} w$. (2) then implies (1).

To prove (6) note that the set $\left(M^{* *} f>\lambda\right)$ is open, so $\left(M^{* *} f>\lambda\right)=\bigcup I_{j}$, where the intervals $I_{j}=\left(\alpha_{j}, \alpha_{j}+\delta_{j}\right)$ are disjoint and $M^{* *} f\left(\alpha_{j}\right) \leqq \lambda$. It is then sufficient to prove

$$
m_{w}\left(x \in I_{j}: M^{* *} f>3 \lambda, H_{r} f \leqq \gamma \lambda\right) \leqq C(\gamma) m_{w}\left(I_{j}\right) .
$$

We may clearly assume there is a point $z_{j} \in I_{j}$ with $H_{r} f\left(z_{j}\right) \leqq \gamma \lambda$.

Let $\bar{I}_{j}=\left(\alpha_{j}-2 \delta_{j}, \alpha_{j}+2 \delta_{j}\right)$,

$$
\begin{aligned}
f_{1}(x) & =f(x), & & x \in \bar{I}_{j}, \\
& =0, & & x \notin \bar{I}_{j},
\end{aligned} \text { and } f_{2}=f-f_{1} .
$$

$m$ will denote Lebesgue measure.

Using (5) we have

$$
\begin{aligned}
m\left(M^{* *} f_{1}>\lambda\right) & \leqq \lambda^{-r} \int\left[M^{* *} f_{1}\right]^{r} \leqq C \lambda^{-r} \int\left|f_{1}\right|^{r} \\
& \leqq C \lambda^{-r}\left[H_{r} f\left(z_{j}\right)\right]^{r} m\left(I_{j}\right) \leqq C \gamma^{r} m\left(I_{j}\right) .
\end{aligned}
$$

For any $x \in I_{j}, n$ and $\varepsilon>0$,

$$
\left|\int_{\varepsilon<|x-t|<\pi} e^{-i n t} f_{2}(t) /(x-t) d t-\int_{\varepsilon<\mid \alpha_{j}-t<\pi} e^{-i n t} f_{2}(t) /(x-t) d t\right|
$$

is majorized by $C_{0} H_{1} f\left(z_{j}\right) \leqq C_{0} H_{r} f\left(z_{j}\right) \leqq C_{0} \gamma \lambda$. It follows that $x \in I_{j}$ implies

and so

$$
M^{* *} f_{2}(x) \leqq M^{* *} f\left(\alpha_{j}\right)+C_{0} \gamma \lambda \leqq\left(1+C_{0} \gamma\right) \lambda
$$

$$
M^{* *} f(x) \leqq M^{* *} f_{1}(x)+M^{* *} f_{2}(x) \leqq M^{* *} f_{1}(x)+\left(1+C_{0} \gamma\right) \lambda .
$$

Hence, $M^{* *} f(x)>3 \lambda, x \in I_{j}$, implies $M^{* *} f_{1}(x)>\lambda$ if $1+C_{0} \gamma<2$. Collecting results we obtain

$$
m\left(x \in I_{j}: M^{* *} f(x)>3 \lambda, H_{1} f \leqq \gamma \lambda\right) \leqq C \gamma^{r} m\left(I_{j}\right) .
$$

(7) follows immediately from (8) and the following consequence of the $A_{p}$ condition:

(9) If $w \in A_{p}$, any $p$, then there are positive constants $C$ and $\delta$ such that 
for any interval $I$ and measurable set $E, m(E \cap I) \leqq \varepsilon m(I)$ implies $m_{w}(E \cap I) \leqq C \varepsilon^{\delta} m_{w}(I)$.

To prove (9) we use the fact that $w \in A_{p}$, any $p$, implies there is $s>1$ and a constant $C$ such that

$$
\left(\int_{I} w^{s}\right)^{1 / s} \leqq C|I|^{(1 / s)-1} \int_{I} w
$$

for all intervals $I$. (See Muckenhoupt [6].) If $(1 / s)+\left(1 / s^{\prime}\right)=1$, Hölder's inequality and (10) imply

$$
\int_{E \cap I} w \leqq(m(E \cap I))^{1 / s^{\prime}}\left(\int_{I} w^{s}\right)^{1 / s} \leqq C(m(E \cap I) / m(I))^{1 / s^{\prime}} \int_{I} w .
$$

This gives (9) and completes our proof.

\section{BIBLIOGRAPHY}

1. D. L. Burkholder and R. F. Gundy, Extrapolation and interpolation of quasilinear operators on martingales, Acta Math. 124 (1970), 249-304.

2. L. Carleson, On convergence and growth of partial sums of Fourier series, Acta Math. 116 (1966), 135-157. MR 33 \#7774.

3. R. R. Coifman, Distribution function inequalities for singular integrals, Proc. Nat. Acad. Sci. U.S.A. 69 (1972), 2838-2839.

4. R. A. Hunt, On the convergence of Fourier series, Orthogonal Expansions and their Continuous Analogues (Proc. Conf., Edwardsville, Ill., 1967), Southern Illinois Univ. Press, Carbondale, Ill., 1968, pp. 235-255. MR 38 \#6296.

5. R. A. Hunt, B. Muckenhoupt and R. Wheeden, Weighted norm inequalities for the conjugate function and Hilbert transform, Trans. Amer. Math. Soc. 176 (1973), 227-252.

6. B. Muckenhoupt, Weighted norm inequalities for the Hardy maximal function, Trans. Amer. Math. Soc. 165 (1972), 207-226. MR 45 \#2461.

Department of Mathematics, Purdue University, West lafayette, Indiana 47907

Current address (W.-S. Young): Department of Mathematics, Northwestern University, Evanston, Illinois 60201 